\title{
Comportamiento sísmico de edificaciones de tapia pisada reforzadas con marcos de madera y viga de coronación en concreto
}

\section{Seismic behaviour of rammed earth buildings reinforced with wood elements and an upper concrete beam}

Cecilia López P. ${ }^{(*)}, \underline{\text { Daniel Ruiz }}^{(* *)}$, Sandra Jerez ${ }^{(* * *)}$, Sandra Aguilar ${ }^{(* * * *)}$, Juan F. Torres ${ }^{(* * * *)}$, Yezid A. Alvarado ${ }^{(* * * * *)}$

\section{RESUMEN}

La construcción en tierra está presente en todo el mundo. Muchas de estas construcciones se encuentran en regiones sísmicas y tienen una vulnerabilidad alta. Con el fin de reducir esta vulnerabilidad se propone una estrategia integrada que consiste en un refuerzo externo con elementos de madera en ambas direcciones y en ambos lados de los muros (en tapia pisada) simultáneamente con la implementación de un diafragma rígido por medio de una viga de coronación en concreto. Se propone que la viga se construya con una transición entre la tierra y el concreto para mejorar la compatibilidad. Los resultados de ensayos en mesa vibratoria en un modelo a escala 1:6 y su contraparte no reforzada muestran una mejora significativa en el comportamiento global de la construcción (reducción en los desplazamientos hasta del 80\% en los modelos reforzados), logrando el objetivo de prevenir el colapso, manteniendo la integridad de la estructura.

Palabras clave: Construcción en tierra, tapia pisada, reforzamiento sísmico, vulnerabilidad sísmica.

\section{ABSTRACT}

Earth construction is present all over the world. Many of these constructions are in seismic regions and have a high seismic vulnerability. To reduce this vulnerability and prevent collapse this paper proposes an integrated strategy consisting in an external reinforcement with wooden elements in both directions and on both sides of the walls (rammed earth) and a rigid diaphragm by means of an upper concrete beam. This beam is proposed to be built with a smooth transition between the earth and the concrete to improve compatibility. The results of shake table tests on a 1:6 scaled model and its unreinforced counterpart show a significant improvement in the global behaviour of the construction (The results of the tests showed a reduction in the displacements up to $80 \%$ in the reinforced models), achieving the main objective of preventing collapse while the structure remains almost intact.

Keywords: Earth construction, rammed earth walls, seismic reinforcement, seismic vulnerability.

(*) Arquitecta. Profesor Asistente. Departamento de Arquitectura, Pontificia Universidad Javeriana, Bogotá (Colombia).

${ }^{* *}$ ) Ingeniero Civil. Director del Departamento de Ingeniería Civil, Pontificia Universidad Javeriana, Bogotá (Colombia).

${ }^{* * *}$ ) Ingeniero Civil. Profesor Asociado. Programa de Ingeniería Civil, Escuela Colombiana de Ingeniería, Bogotá (Colombia).

$\left(^{* * * *}\right)$ Ingeniero Civil. Profesor Asistente. Programa de Ingeniería Civil, Escuela Colombiana de Ingeniería, Bogotá (Colombia).

(****) Ingeniero Civil. Profesor Instructor. Programa de Ingeniería Civil, Escuela Colombiana de Ingeniería, Bogotá (Colombia).

(*****) Ingeniero Civil. Jefe del Laboratorio de Pruebas y Ensayos, Pontificia Universidad Javeriana, Bogotá (Colombia).

Persona de contacto/Corresponding author: daniel.ruiz@javeriana.edu.co (D. Ruiz).

ORCID: https://orcid.org/oooo-0002-3974-4192 (C. López); https://orcid.org/oooo-0003-4164-0357 (D. Ruiz);

https://orcid.org/oooo-0002-9646-4621 (S. Jerez); https://orcid.org/oooo-0002-0877-8752 (S. Aguilar);

https://orcid.org/oooo-0001-8518-8855 (J. Torres); https://orcid.org/oooo-0002-1260-8211 (Y. Alvarado).

Cómo citar este artículo/Citation: López P., Cecilia; Ruiz, Daniel; Jerez, Sandra; Aguilar, Sandra; Torres, Juan F.; Alvarado, Yezid A. (2020). Comportamiento sísmico de edificaciones de tapia pisada reforzadas con marcos de madera y viga de coronación en concreto. Informes de la Construcción, 72(559): e347. https://doi.org/10.3989/ic.70914.

Copyright: (c) 2020 CSIC. Este es un artículo de acceso abierto distribuido bajo los términos de la licencia de uso y distribución Creative Commons Reconocimiento 4.0 Internacional (CC BY 4.0). 


\section{INTRODUCCIÓN}

Uno de los grandes problemas que afronta la humanidad actualmente es el déficit de vivienda para la población con menores ingresos, siendo uno de los mayores retos a nivel mundial ya que tiene implicaciones sociales, económicas y culturales.

Desde 1948, la Declaración Universal de Derechos Humanos determinó el "derecho al techo", que luego fue corroborado por la Conferencia de las Naciones Unidas en 1976 y 1996 (1). Para 1998 (2) se reportaba que, para mitigar el déficit habitacional en América Latina, era necesario construir cuatro millones de viviendas por año durante toda una generación. El mismo documento reportaba que solo se llegó a construir dos millones de viviendas por año, con lo cual el problema continúa creciendo de forma exponencial.

Como respuesta a este déficit y al derecho a la vivienda que tiene la población en distintos hemisferios, se han buscado diversas alternativas a nivel económico, espacial, constructivo y tecnológico que permitan mitigar y ampliar la cobertura de techo a las familias más necesitadas.

Dentro del ámbito tecnológico se han explorado diferentes alternativas de materiales de construcción como desechos industriales, desechos vegetales post cosecha y la reincorporación de materiales y técnicas vernáculas. Una de las más destacadas, de mayor difusión y uso son las realizadas con tierra cruda.

La tierra ha sido desde los inicios de la humanidad uno de los materiales más utilizados como material de construcción, dada su abundancia y disponibilidad. En todos los continentes se encuentran edificaciones patrimoniales en tierra, siendo este material todavía empleado, en la actualidad, como material de construcción para viviendas nuevas. En el contexto actual, la tierra constituye una opción con numerosas ventajas, pues no requiere de transporte, ya que se puede extraer del sitio y en su proceso de producción no tiene procesos contaminantes o de emisión de gas carbónico. De hecho, es considerada como un material de construcción viable, durable y sostenible (3).

En Colombia, las construcciones en tierra son parte fundamental del patrimonio cultural; se encuentran aproximadamente 1118 monumentos declarados como patrimoniales, de los cuales el 90\% están hechos en tierra tal como lo reportan Lopez et al. (4).

Las técnicas propias de la construcción en tierra han evolucionado en la medida en que se ha evidenciado la vulnerabilidad de este tipo de construcciones frente a fenómenos que van desde aquellos asociados al clima (humedad excesiva, altas temperaturas) hasta los que son producto de fenómenos naturales extremos como sismos e inundaciones. Es así como países como Perú o Chile han desarrollado normas de construcción en tierra para tipologías específicas, reglamentando su uso en función de la zonificación sísmica y estableciendo límites y recomendaciones que garanticen un comportamiento adecuado frente a sismos y por supuesto frente a cargas verticales $(5,6)$.

El desarrollo de construcciones en tierra se ha dado prácticamente en todos los continentes, por lo que el problema de su conservación se ha abordado también en todo el mundo. Sin embargo, en zonas con amenaza sísmica intermedia y alta se ha hecho énfasis en el reforzamiento dada su alta vulnerabilidad frente a sismos. Los estudios muestran que estas estructuras son altamente vulnerables frente a fuerzas sísmicas debido a la manera en que están construidas. Esta vulnerabilidad proviene de características propias de la configuración y de factores medio ambientales.

En cuanto a los problemas de configuración se encuentran irregularidades en planta y en altura, grandes aberturas sin refuerzo en los muros, falta de amarre entre los muros al nivel de cubierta, insuficiente área de muros en las direcciones principales de la estructura para soportar solicitaciones de corte y defectos en los cimientos, entre otros. A todo esto se le suma por un lado, la gran masa que suelen soportar estas edificaciones debido a la configuración de las cubiertas (sumado al peso propio de los muros), y por otro, el efecto de las cargas dinámicas propias de un sismo que tienden a desagregar tanto el muro como el material mismo al afectar directamente la cohesión, principal fuerza de soporte de la tierra cruda, tal como lo reportan los autores AIS (7), Papanikolau y Taucer (8), Yamin et al. (9) y Blondet et al. (10).

Es así como sobre el comportamiento de edificaciones de tierra hay consenso en que los puntos débiles al verse éstas sometidas a fuerzas laterales son: i) los esfuerzos de tracción que se generan en los muros dentro y fuera del plano y que el material es incapaz de soportar, ii) la integridad de las esquinas que no se mantiene, iii) la ausencia de un diafragma rígido al nivel de la cubierta, iv) la conexión deficiente entre los muros y el sistema de cubierta, v) la baja resistencia fuera del plano de los muros y vi) el peso de las cubiertas que caen sobre los muros haciéndolos fallar. Lo anterior lo reportan López et al. (4), Blondet et al. (10), Yamín et al. (11), Tolles y Krawinkler (12), Ruiz et al. (13) y Ruiz et al. (14).

Estas características usualmente encontradas en este tipo de construcciones llevan al agrietamiento vertical de los muros, la separación entre los componentes del muro, la desagregación del material y finalmente el colapso de los muros, que habiendo perdido buena parte de su capacidad de carga ya no pueden soportar las cargas verticales.

\subsection{Estrategias de sistemas de refuerzo para edificaciones en tierra}

Las estrategias de reforzamiento, como en otros sistemas estructurales, se concentran en proveer a los muros y al sistema completo las propiedades necesarias para soportar un sismo: rigidez, resistencia y capacidad de ductilidad al desplazamiento, entendiéndose por soportar el sismo, evitar el colapso minimizando el número de víctimas y conservando el patrimonio histórico (en el caso del refuerzo de edificaciones existentes). Algunas estrategias han buscado estas propiedades reforzando los muros en su interior, añadiendo refuerzo longitudinal y transversal de diferentes materiales. Otros autores (como Silveira et al. (15) y Parisi et al. (16)) han trabajado sobre la implementación de elementos externos de reforzamiento que deben trabajar en conjunto con el muro, esencialmente confinándolo, aumentando su rigidez lateral y modificando el comportamiento del conjunto.

De acuerdo con Blondet et al. (10) y con Gómez et al. (17), cualquier estrategia de reforzamiento sobre muros en tierra debe implementarse por ambas caras del muro y de forma continua pues los esfuerzos de tracción responsables de la falla de los muros pueden presentarse en cualquier parte. Igualmente, los elementos de refuerzo deben estar bien integrados al muro y protegidos del medio ambiente. 
Pasando a las estrategias de refuerzo externo se encuentra que son numerosas y diversas. Algunas de estas son: pañete con malla electro soldada y mortero, pañete con malla plástica y mortero, franjas de malla de corrales y mortero, entramados en madera, elementos de concreto reforzado, tensores de acero para confinamiento, inclusión de elementos en piedra y combinaciones de una o varias de las anteriores. Las alternativas descritas anteriormente pueden encontrarse en (4, 9, 10, 18, 19, 20). A continuación se presentarán detalles de tres de las principales alternativas de rehabilitación: malla y pañete, refuerzo con madera e implementación de un diafragma rígido.

\subsection{Reforzamiento con malla y pañete}

Esta propuesta consiste en colocar malla electro soldada en ambas caras del muro en franjas horizontales y verticales en las zonas críticas de la estructura, simulando el efecto de vigas y columnas de confinamiento, como se muestra en la Figura 1a. La malla se une al muro mediante clavos y tapas de gaseosa o arandelas cada $30 \mathrm{~cm}$. Se utilizan conectores que unen el refuerzo interno y externo cada $50 \mathrm{~cm}$. Los conectores se componen de un alambre de $8 \mathrm{~mm}$ que se coloca en orificios previamente perforados, los cuales posteriormente se rellenan con mortero de cal y arena en proporción 1:2. Se debe tener especial cuidado en colocar acero en todos los sitios donde se pueden concentrar los esfuerzos de corte y los esfuerzos de flexión, como en los vanos de puertas y ventanas o en las esquinas tal como se presenta en la Figura 1b (9).
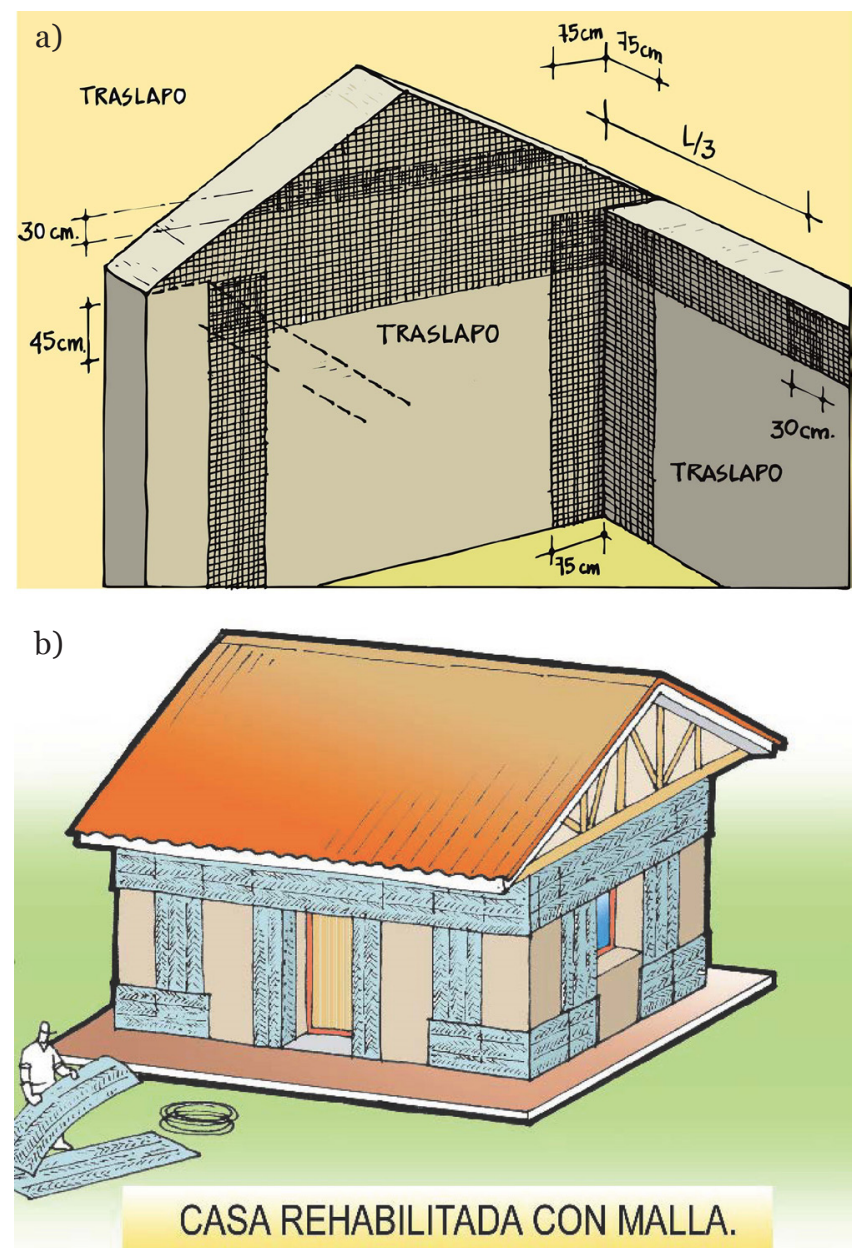

Figura 1. a) Instalación de la malla de refuerzo, b) Estructura reforzada. Fuente: Imagen tomada de (7).
Los autores Ruiz et al. (13) y Yamín et al. (11) realizaron ensayos de mesa vibratoria en modelos a escala reforzados con malla de acero con mortero de cal por una sola cara (la externa), como complemento a los ya mencionados en el sistema de reforzamiento con madera y con las mismas características de medición. Los resultados obtenidos por los autores Ruiz et al. (13) y Yamín et al. (11) sugirieron que este sistema reduce la fisuración y el agrietamiento y disminuye los desplazamientos de los muros. Como resultado se obtuvo que este sistema de refuerzo permite mantener la integridad estructural de la estructura, pero no funciona tan bien como los entramados de madera que se explicarán a continuación (aparecen más fisuras y hay mayores desplazamientos relativos mayores al reforzar con mallas).

\subsection{Refuerzo con madera}

Esta técnica propuesta inicialmente por Yamín et al. (11), consiste en colocar elementos de madera de $200 \mathrm{~mm}$ de ancho y entre 20 y $30 \mathrm{~mm}$ de espesor, en las dos caras del muro e interconectarlos mediante pernos pasantes. Se instalan maderas tanto horizontales como verticales separadas una distancia de $1500 \mathrm{~mm}$. Es deseable instalar maderas adicionales en las esquinas y en los vanos de puertas y ventanas. En las uniones de esquina deben instalarse platinas de acero para darle continuidad a los anillos horizontales, como se ve en la Figura 2. Estas platinas a su vez deben estar ancladas a los muros y a los elementos de madera.

a)

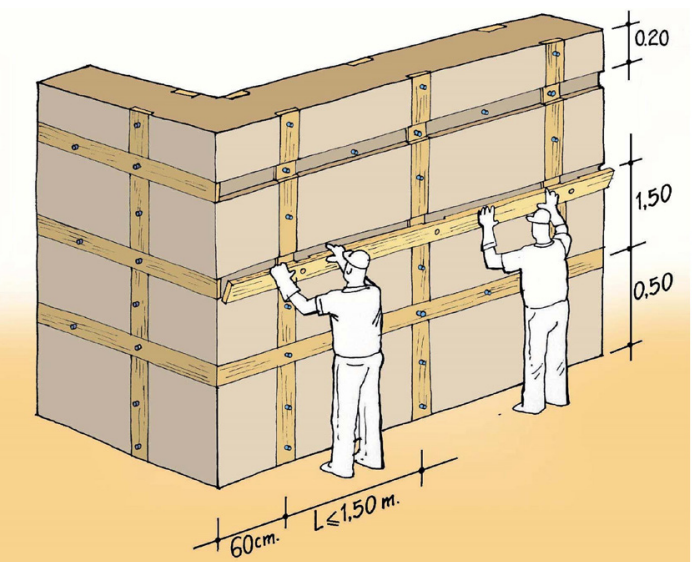

b)

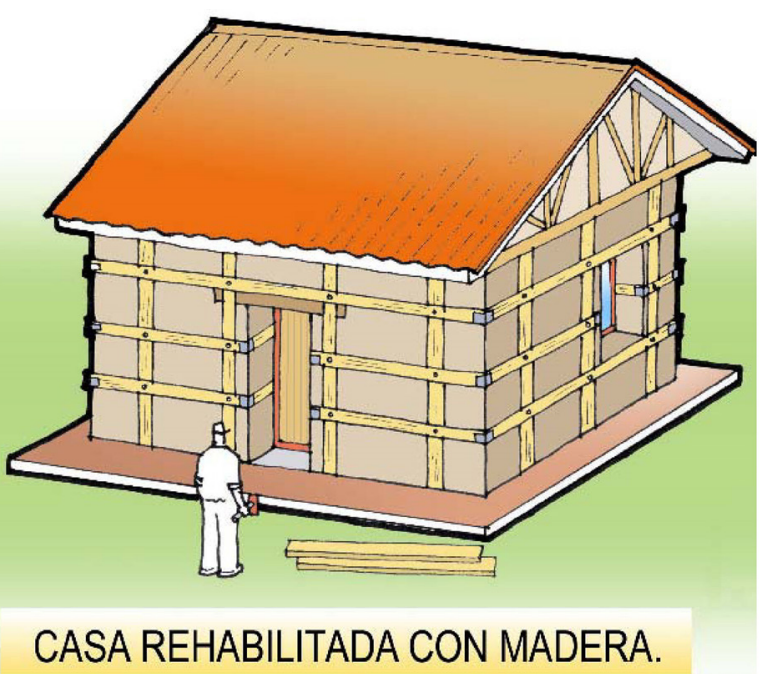

Figura 2. a) Instalación de un entramado de madera, b) Estructura reforzada. Fuente: Imagen tomada de (7). 
Para garantizar la compatibilidad de deformaciones, se colocan conectores cada cierta longitud y para proporcionar una superficie de contacto rugosa, se clavan puntillas sobre las tablas. Este reforzamiento incrementa la resistencia a flexión de los muros, mantiene la unidad de la estructura y brinda una mayor capacidad de disipación de energía. Varios estudios reportan una mejora en el comportamiento de los muros ante cargas paralelas al plano del mismo, incrementando el desplazamiento último (hasta en 400\%) y el esfuerzo cortante promedio resistente (hasta en 100\%). Lo anterior es reportado por Yamín et al. (11), Ruiz et al. (13), Ruiz et al. (14) y por Gómez et al. (17).

\subsection{Implementación de un diafragma rígido}

Una de las principales causas de la vulnerabilidad en construcciones de tierra durante eventos sísmicos es la ausencia de un diafragma rígido causando que los muros actúen como elementos individuales debilitándose en las esquinas y fallando entre ellos. La implementación de un diafragma rígido es por lo tanto necesaria para garantizar que la edificación se comportará adecuadamente durante el evento sísmico y reduce la probabilidad de falla en las esquinas. En Colombia, diversos inmuebles patrimoniales en tierra han sido rehabilitados mediante sistemas de diafragmas y vigas hechas de madera y concreto. Estas alternativas han resultado en mejoras en el desempeño global. Sin embargo, la mayor preocupación acerca de este sistema es la compatibilidad entre ambos materiales (concreto-tierra o madera-tierra) en la conexión viga-muro debido a que sus propiedades mecánicas e incluso su rigidez son bastante diferentes. Esta técnica de reforzamiento con una transición desde el suelo hasta el concreto se ha implementado en Colombia por el Ing. Luis Fernando Velasco, en las ciudades de Popayán y Bogotá, en varias edificaciones existentes de tierra (Iglesia La Candelaria e Instituto Caro y Cuervo en la ciudad de Bogotá; Iglesia de San José en la ciudad de Popayán).

\section{METODOLOGÍA: PROPUESTA DE REFORZAMIENTO EN MADERA CON TRANSICIÓN PARA VIGA DE CORONACIÓN EN CONCRETO}

Con base en lo anterior este trabajo propone combinar el reforzamiento de muros con entramados de madera con una estrategia de mejoramiento del diafragma. Esto con el fin de proporcionar un confinamiento en los muros que garantice la integridad de la estructura después del movimiento y a la vez lograr un comportamiento de conjunto y una adecuada distribución de las fuerzas sísmicas en función de la rigidez relativa de los muros.

En algunos ensayos se ha evidenciado que la diferencia de rigidez entre la viga de coronación y el muro genera una superficie de falla frente a fuerzas horizontales (4). Por esta razón se propone aquí hacer una transición en cuatro capas variando el contenido
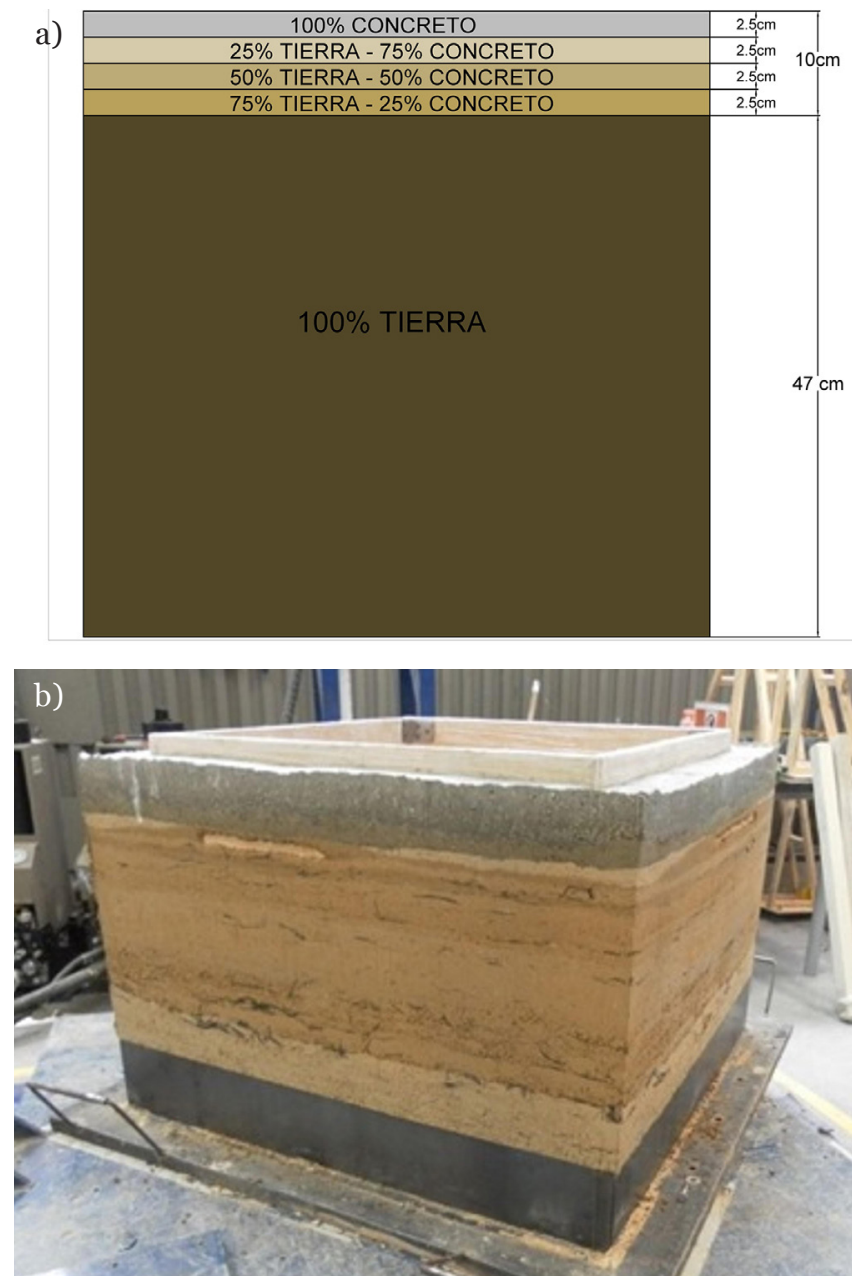

Figura 3. a) Composición de la transición entre el muro y la viga de concreto y b) Modelo de tapia pisada construido con la transición propuesta. Fuente: Autores.

de tierra y de cemento de abajo hacia arriba así: 75\%-25\%, 50\%$50 \%, 25 \%-75 \%$ y $100 \%$ de mortero, véase la Figura 3. Esta zona de transición tiene una altura cercana al 20\% de la dimensión vertical muro (incluyendo la zona de 100\% mortero); y un espesor igual al del muro. Así mismo, es importante anotar que el elemento de refuerzo de madera horizontal superior se ancló en la zona de $25 \%$ Tierra $-75 \%$ mortero con el fin de mejorar la conexión viga-muro-refuerzo. Los autores determinaron que era viable ubicar dicho anclaje del último elemento horizontal en esta penúltima zona de transición antes de la capa de 100\% mortero, aunque por supuesto también es viable ubicar dicho anclaje en la capa del 100\% de mortero (sin tierra).

En la Tabla 1 se presentan las proporciones (en peso) de los materiales usados en cada una de las mezclas de las diferentes zonas de transición.

Tabla 1. Proporciones (en peso) de los materiales usados en la zona de transición.

\begin{tabular}{|c|c|c|c|c|c|c|c|c|c|c|}
\hline Material & \multicolumn{2}{|c|}{ 100\% Tierra } & \multicolumn{2}{|c|}{ 75\% Tierra } & \multicolumn{2}{|c|}{ 50\% Tierra } & \multicolumn{2}{|c|}{ 25\% Tierra } & \multicolumn{2}{|c|}{ 100\% Mortero } \\
\hline Arcilla & \multirow{4}{*}{$100 \%$} & $4.0 \%$ & \multirow{4}{*}{$75 \%$} & $3.0 \%$ & \multirow{4}{*}{$50 \%$} & $2.0 \%$ & \multirow{4}{*}{$25 \%$} & $1.0 \%$ & \multirow{3}{*}{ o\% } & $0.0 \%$ \\
\hline Cal & & $1.0 \%$ & & $0.7 \%$ & & $0.5 \%$ & & $0.3 \%$ & & $0.0 \%$ \\
\hline Tierra & & $84.0 \%$ & & $63.0 \%$ & & $42.0 \%$ & & $21.0 \%$ & & $0.0 \%$ \\
\hline Agua & & $11.0 \%$ & & $8.3 \%$ & & $5.5 \%$ & & $2.8 \%$ & \multirow{3}{*}{$100 \%$} & $8.9 \%$ \\
\hline Cemento & \multirow{2}{*}{ o\% } & $0.0 \%$ & \multirow{2}{*}{$25 \%$} & $5.5 \%$ & \multirow{2}{*}{$50 \%$} & $10.9 \%$ & \multirow{2}{*}{$75 \%$} & $16.4 \%$ & & $21.8 \%$ \\
\hline Arena & & $0.0 \%$ & & $19.5 \%$ & & $39.1 \%$ & & $58.6 \%$ & & $69.4 \%$ \\
\hline
\end{tabular}




\subsection{Descripción de los modelos a escala para ensayo en mesa vibratoria}

Con el fin de ensayar la estrategia propuesta, se construyeron dos modelos a escala reducida: uno sin reforzamiento o viga superior, y otro con la transición mencionada en la viga superior y reforzamiento en madera. Ambos modelos estaban en una escala 1:6 de una vivienda representativa de un piso y $36 \mathrm{~m}^{2}$ de área en planta (véase la Figura 4), que es el tamaño mínimo establecido para viviendas de interés social (VIS) en Colombia. Las dimensiones en planta de cada modelo eran de $1 \mathrm{~m}$ x $1 \mathrm{~m}$ y su altura era de $57 \mathrm{~cm}$. El modelo contaba con 4 muros con espesor de $8 \mathrm{~cm}$ : uno completo y los otros tres con aberturas para ventanas y puerta. Con el fin de analizar el efecto de la localización de las aberturas, uno de los muros tenía la ventana en el centro y el otro en el borde del muro, cerca de la esquina. Finalmente, el muro restante tenía una puerta central. La cara que cerraba el cuadrado no tenía ninguna abertura. El techo fue diseñado como una cercha de madera donde se ubicaron varias piezas metálicas para simular la masa del techo. La cimentación se construyó sobre una formaleta metálica con una mezcla de piedra, arena y arcilla, con el mismo espesor de los muros, (véase la Figura 5).

El reforzamiento en madera estaba compuesto por elementos verticales y horizontales de $35 \mathrm{~mm}$ (200 mm a escala real) de ancho y $5 \mathrm{~mm}$ (30 $\mathrm{mm}$ a escala real) de espesor, separados $250 \mathrm{~mm}$ ( $1500 \mathrm{~mm}$ a escala real) en ambas direcciones, de acuerdo con las recomendaciones del "Manual para la rehabilitación de viviendas construidas en adobe y tapia pisada" de la referencia (7). En las esquinas los elementos de madera se conectaron con ángulos de acero atornillados. La transición entre el muro y la viga superior fue de $10 \mathrm{~cm}$ de espesor con 4 capas de $2.5 \mathrm{~cm}$ cada una, (véase la Figura 3a).
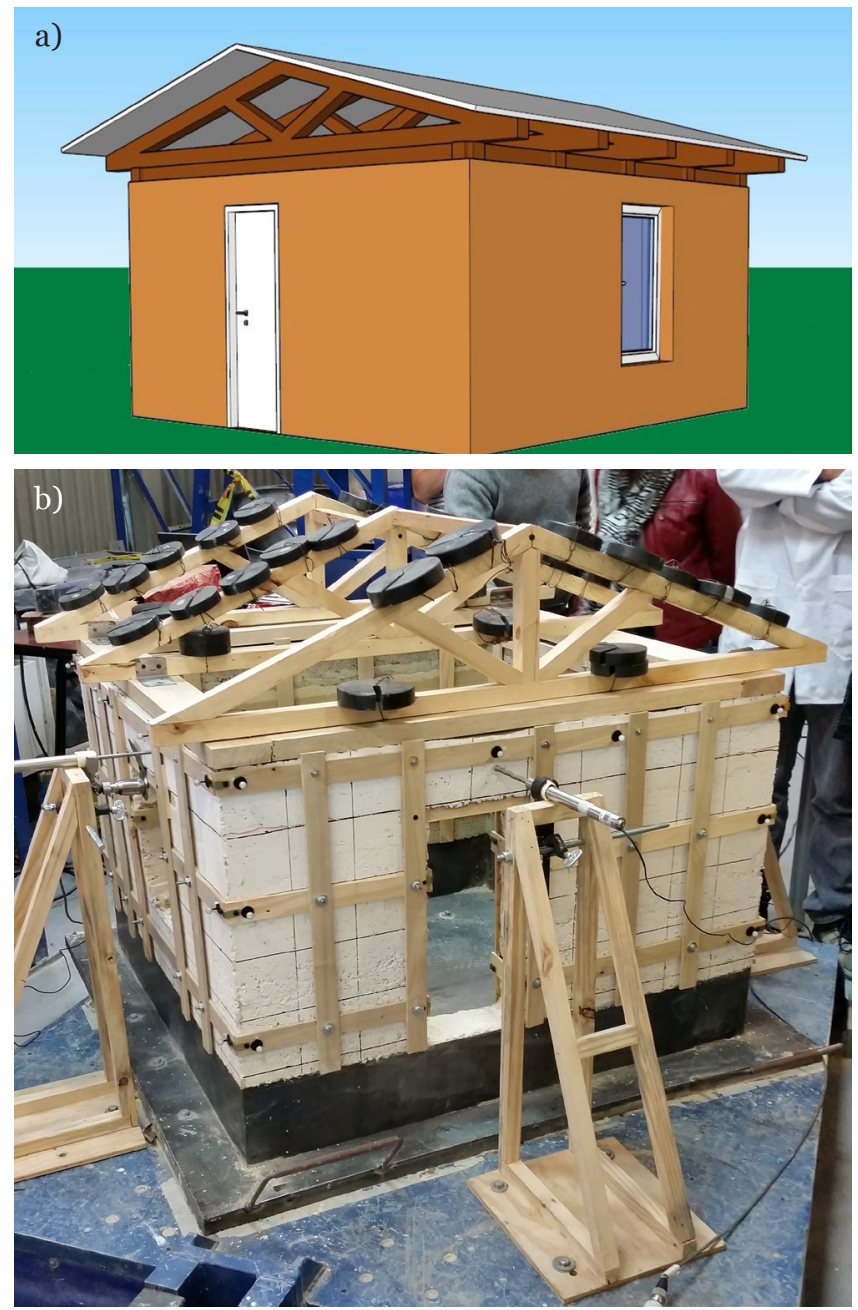

Figura 5. a) Esquema del modelo sin refuerzo y b) Modelo reforzado. Fuente: Autores.
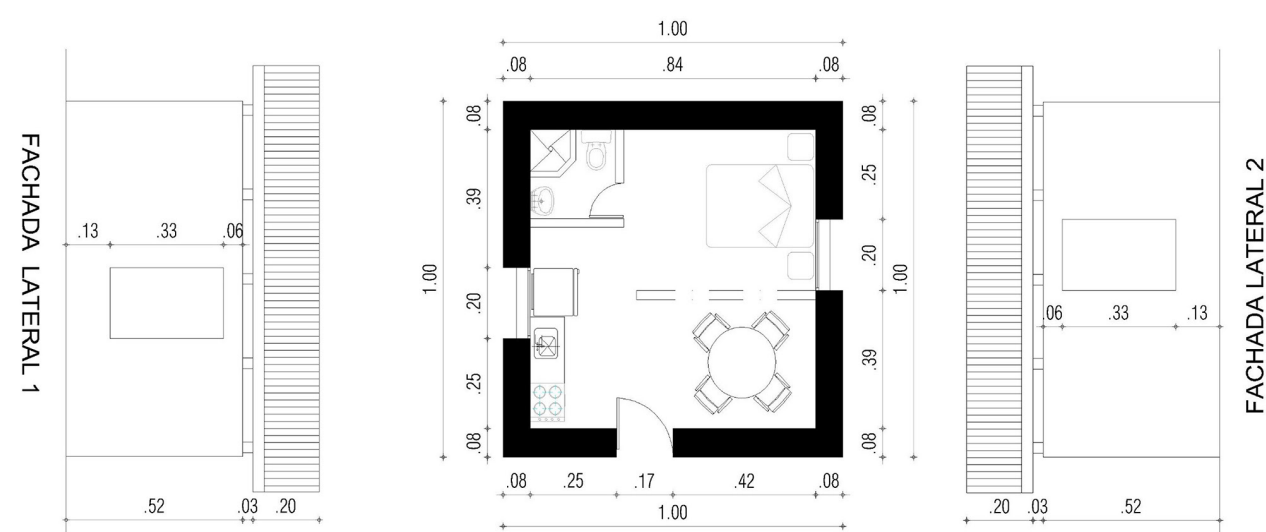

PLANTA GENERAL

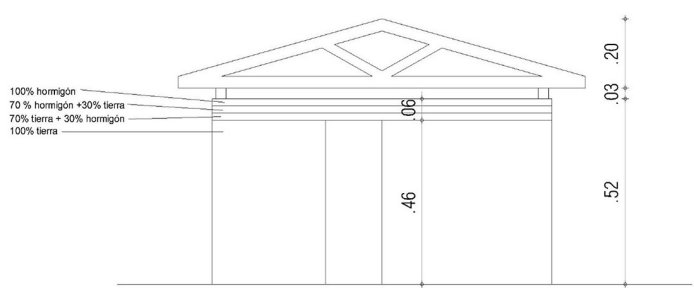

FACHADA PRINCIPAL

Figura 4. Planta y cortes del modelo representativo de una vivienda de $36 \mathrm{~m}^{2}$. Fuente: Autores. 
Estos modelos se ensayaron en una mesa vibratoria unidireccional (1.5 m x $1.5 \mathrm{~m}$ ) controlada por un actuador dinámico MTS con capacidad de $100-\mathrm{kN}$ de carga y un desplazamiento máximo total de $250 \mathrm{~mm}$, que puede generar aceleraciones superiores a $5.0 \mathrm{~g}$, soportando modelos estructurales de hasta $15 \mathrm{kN}$. Los modelos se sometieron a un registro de movimiento fuerte representativo de la amenaza de Bogotá, el del sismo de Tauramena del 19 de enero de 1995 (sismo usado por varias de las investigaciones en tierra en Colombia). Este registro fue escalado y la escala del eje correspondiente al tiempo fue modificada para ajustarlo al tamaño de los modelos ensayados. Los niveles de aceleración se incrementaron basándose en las recomendaciones de Harris et al. (21). La señal original se muestra en la Figura 6 a y la de desplazamiento vs tiempo escalada se muestra en la Figura 6b.

Con el fin de reproducir la excitación sísmica en las dos direcciones principales, los modelos se ensayaron rotados $45^{\circ}$ en planta. La instrumentación de los modelos consistió en dos acelerómetros (dos sobre los modelos y uno registrando las aceleraciones de la mesa vibratoria), cuatro LVDTs (Load Variable Displacement Transducers) y un conjunto de cámaras colocadas de manera que podían registrar desplazamientos y aceleraciones en cada eje principal del modelo, en cada muro y en cada eje de la mesa vibratoria, (véase la Figura 7).

\section{RESULTADOS Y DISCUSIÓN}

\subsection{Comportamiento global}

Con respecto a los modelos construidos, el comportamiento global de ambas estructuras fue bastante diferente, tal como se esperaba. El modelo sin reforzar no resistió la señal entera ya que a los 16 segundos todos los muros presentaron fallas locales seguidas de un colapso parcial y aproximadamente a los 20 segundos toda la estructura colapsó, como se muestra en la Figura 8a. Por su parte, el modelo reforzado resistió
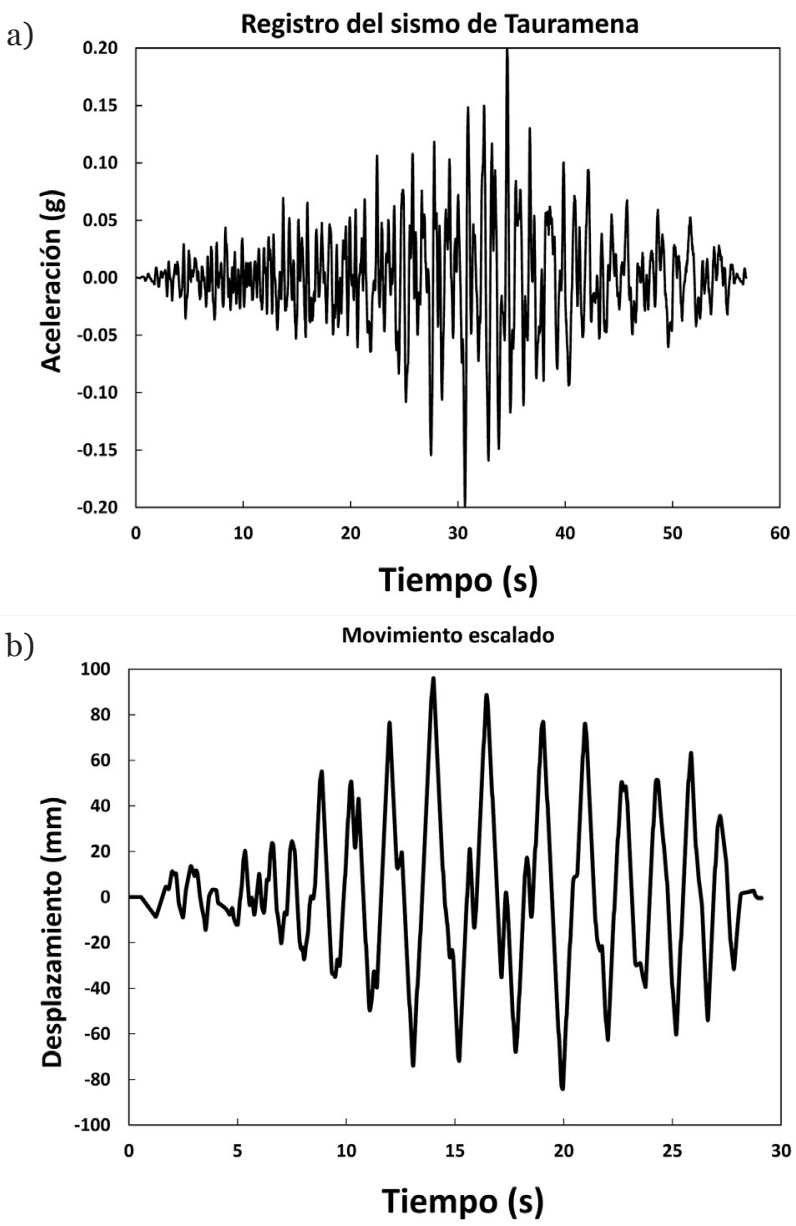

Figura 6. a) Registro del terremoto de Tauramena y b) Movimiento en la base, escalado para los ensayos.

el movimiento en la base por completo con tan solo algunas fisuras en las zonas inferiores, cerca de las esquinas de la cimentación, y también cerca al primer elemento horizontal, (véase la Figura 8b).
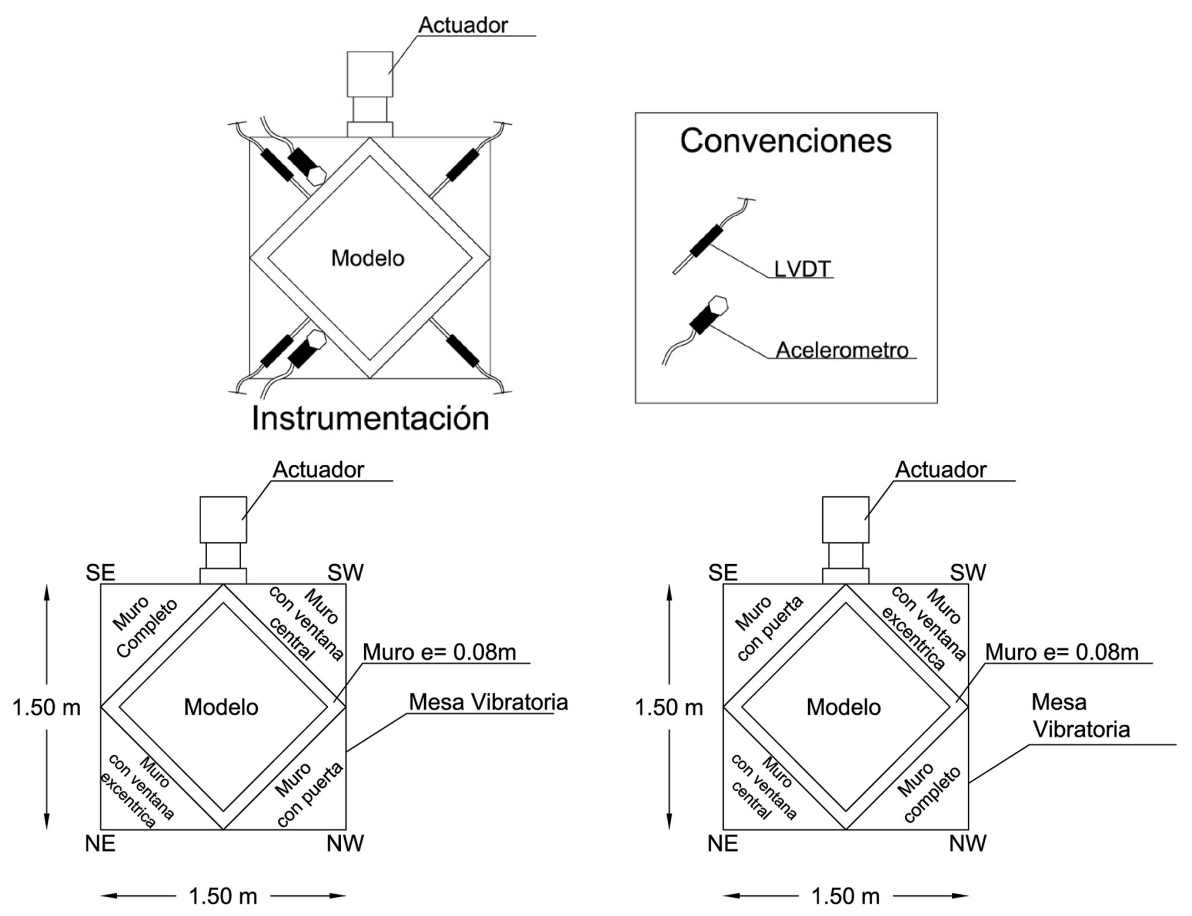

Figura 7. Esquema de instrumentación de los modelos. 

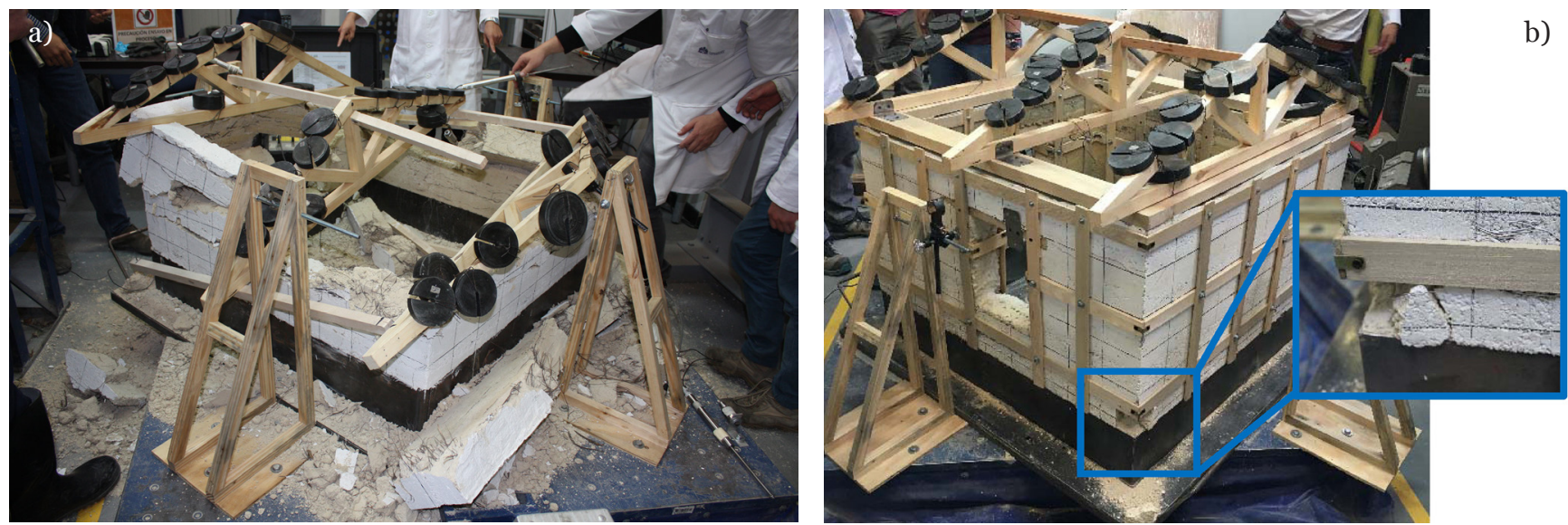

Figura 8. Comportamiento global de: a) modelo sin refuerzo después del ensayo, y b) modelo reforzado después del ensayo. Fuente: Autores.

\subsection{Respuesta de desplazamientos}

Se encontró que los desplazamientos laterales se redujeron entre $65 \%$ y $80 \%$ cuando se compararon los datos de los modelos reforzado y sin refuerzo. La Tabla 2 muestra las reducciones de desplazamientos en cada muro y en la Figura 9 se compara la historia de desplazamientos para uno de los muros (el que tenía una abertura central para ventana). Los otros muros se comportaron de manera similar y no se encontraron desplazamientos permanentes después de la aplicación de la señal de movimiento fuerte.

Como cada modelo fue ensayado en sus dos direcciones principales, todos los muros fueron sometidos a flexión y corte dentro y fuera del plano. Del comportamiento se puede decir que el mecanismo de falla para el modelo sin refuerzo es una combinación de fallas dentro y fuera del plano de los muros iniciado por fisuras de flexión y corte propagándose desde las aberturas hasta la región exterior de cada muro. También, como se esperaba, la falta de solidez en las esquinas fue también una de las causantes de fallas.

Con respecto al modelo reforzado, no se presentó colapso después de haber sido sometido al mismo movimiento fuerte. La región más frágil pareció ser aquella entre la cimentación y la primera línea de refuerzo horizontal. Las fisuras horizontales se observaron justo entre la cimentación y la primera línea de refuerzo horizontal. Como consecuencia, la parte inferior de una de las esquinas se separó del modelo en forma de cuña, (véase la Figura 8b). A pesar de esto, la integridad de la estructura se mantuvo después del movimiento.

\subsection{Comportamiento de la transición concreto-tierra}

Con el fin de evaluar la transición propuesta para mejorar la compatibilidad entre el material de la viga de coronación y

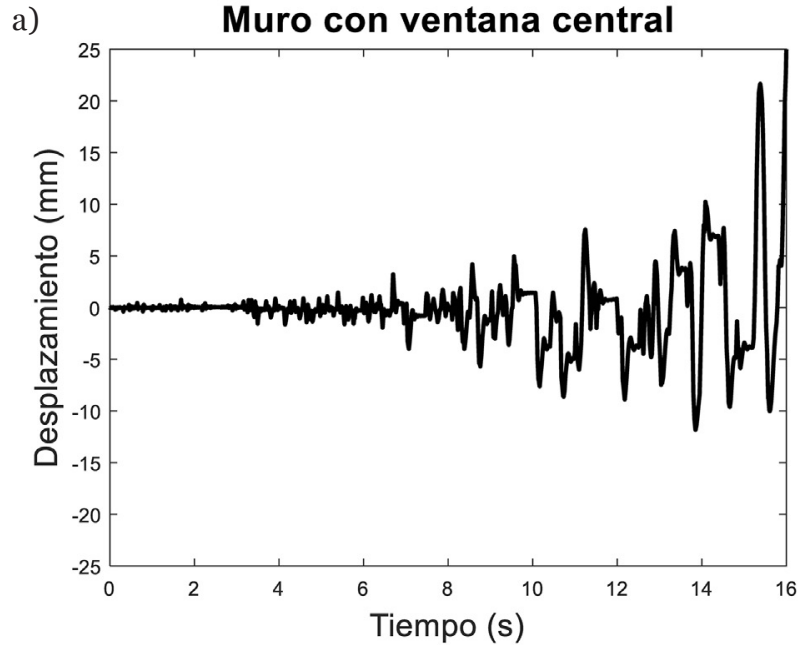

b) Muro con ventana central reforzado

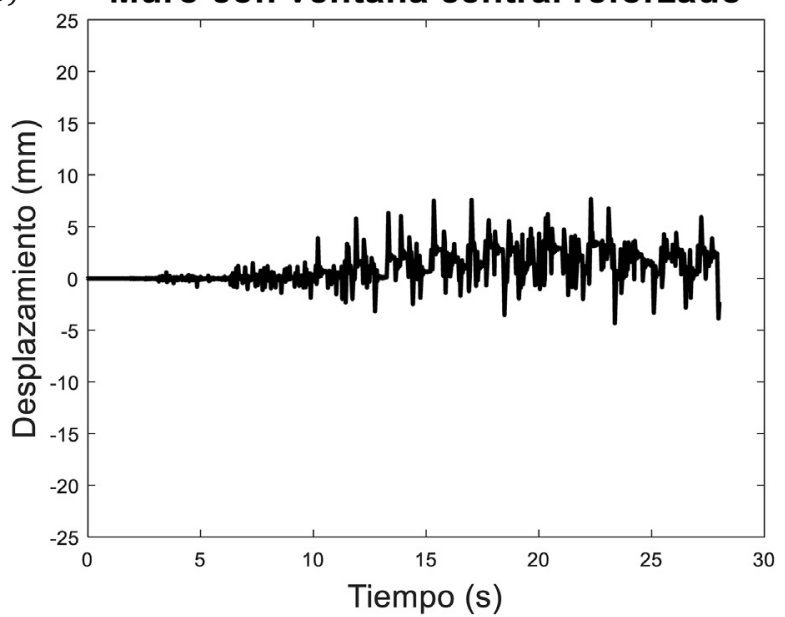

Figura 9. Respuesta de desplazamiento para el muro con ventana central, sin refuerzo y con el refuerzo propuesto.

Tabla 2. Comparación de los máximos desplazamientos fuera del plano para cada muro de la edificación.

\begin{tabular}{|l|c|c|c|}
\hline \multirow{2}{*}{ Muro } & \multicolumn{2}{c|}{ Máximos desplazamientos fuera del plano del muro (mm) } \\
\cline { 2 - 4 } & Modelo sin refuerzo & Modelo reforzado & Reducción (\%) \\
\hline Muro completo & 19.4 & 5.64 & 70.6 \\
\hline Muro con abertura central (ventana) & 24.6 & 7.67 & 68.8 \\
\hline Muro con abertura excéntrica (ventana) & 24.0 & 7.53 & 68.6 \\
\hline Muro con abertura para puerta & 26.4 & 5.68 & 78.5 \\
\hline
\end{tabular}



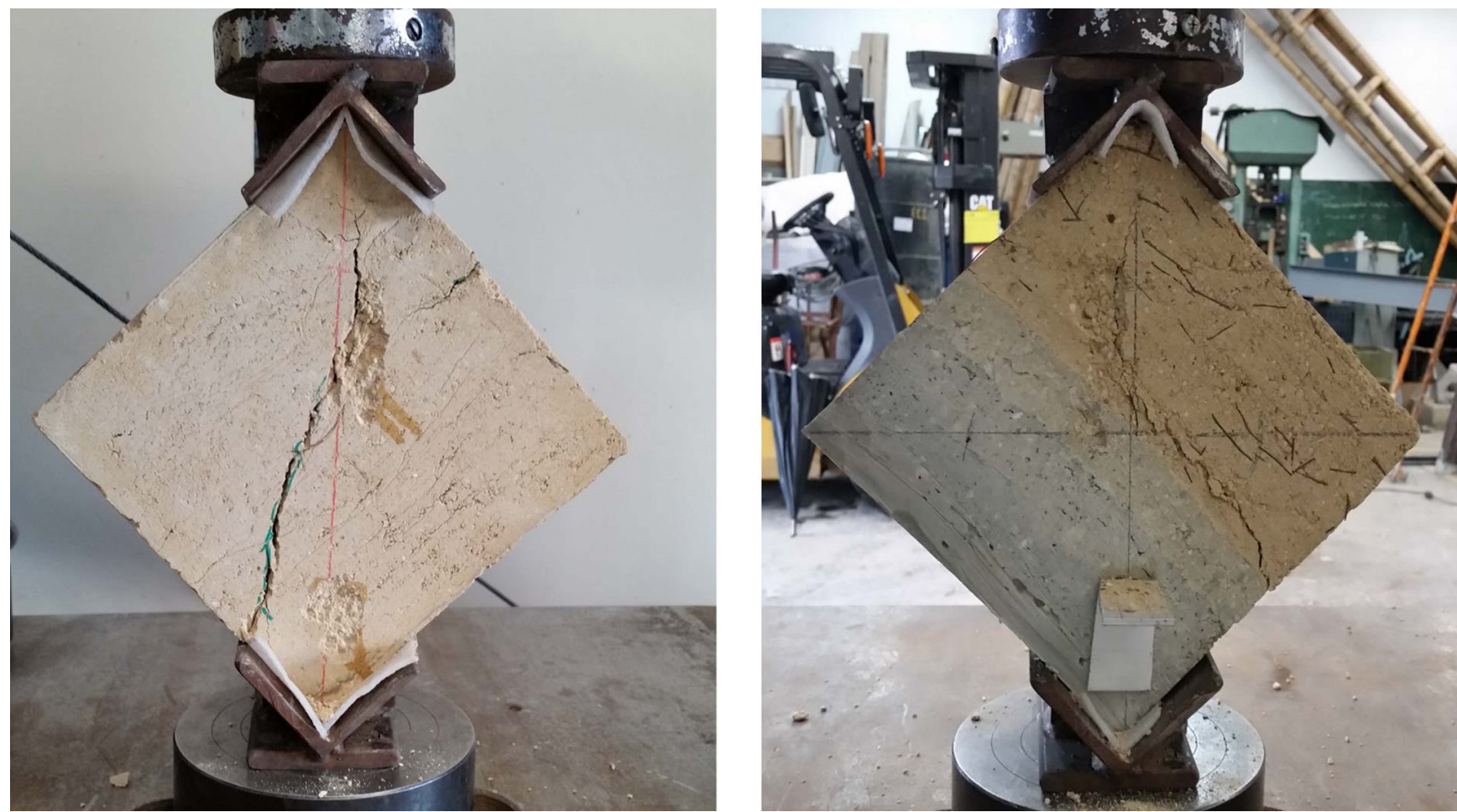

Figura 10. Ensayo de compresión diagonal, sin refuerzo y con la transición tierra-concreto propuesta.

el del muro, se hicieron ensayos de compresión diagonal de acuerdo con la norma ASTM E 519 en especímenes sólo de tierra y de tierra con transición. Los resultados indican un aumento en la resistencia al corte de $59 \%$ en el espécimen con transición y una buena integración entre ésta y el muro, ya que la falla se presenta verticalmente hasta que se encuentra un material más resistente, como se muestra en la Figura 10 y a partir de ahí, tiende a seguir un camino paralelo a la interface, pero no se presenta en esa superficie. Así, la transición cumple un propósito de mejorar la compatibilidad entre el muro y la viga de coronación.

\section{CONCLUSIONES}

En este trabajo se propuso una solución para el reforzamiento de viviendas en tapia pisada, utilizando las ventajas de dos estrategias ya conocidas: el refuerzo externo con entramados de madera y la implementación de una viga de coronación en concreto. El objetivo primario fue reducir la vulnerabilidad de este tipo de construcción con la implementación de un diafragma rígido y el reforzamiento de los muros, que consistía en entramados de madera unidos por ambas caras, dando de esta forma confinamiento y rigidez.

Se encontraron resultados prometedores después del ensayo en mesa vibratoria de modelos a escala reducida (1:6) con y sin refuerzo, sometidos a un movimiento sísmico real. Los resultados mostraron una reducción en los desplazamientos entre $65 \%$ y $80 \%$. Así mismo se redujo la fisuración global, ya que el modelo sin refuerzo tuvo un colapso completo mientras que el modelo reforzado tuvo tan sólo dos fisuras apreciables. Se encontró que el comportamiento general de la edificación mejora sustancialmente con respecto a lo reportado para el reforzamiento sólo con entramados de madera, en donde se evita el colapso, pero los muros sufren agrietamiento importante. Esto podría significar que a escala real se podría garantizar la protección de la vida humana a la vez que no hay una pérdida del patrimonio.

\section{TRABAJOS FUTUROS}

Para futuros trabajos, se buscará determinar el aporte del refuerzo en madera y de la viga de coronación por separado. Así mismo, se buscará optimizar los esquemas de reforzamiento de tal manera que se utilicen menos madera y menos concreto, produciendo las mismas mejoras en comportamiento del sistema.

\section{AGRADECIMIENTOS}

Este trabajo fue posible gracias a la financiación por los fondos de investigación de la Escuela Colombiana de Ingeniería Julio Garavito y la Pontificia Universidad Javeriana (Título del proyecto: Comportamiento sísmico de viviendas construidas en tapia pisada, reforzadas con maderas de confinamiento y con un diafragma rígido. ID Propuesta: 00007069, Id Proyecto: 7201). Asimismo, los autores agradecen a la Vicerrectoría de investigación y los Departamentos de Ingeniería Civil y Arquitectura de la Pontificia Universidad Javeriana por el apoyo para el desarrollo del proyecto. 


\section{BIBLIOGRAFÍA}

(1) PNUD - UN Hábitat. (2004). Hábitat y desarrollo humano. Cuadernos PNUD-UN Hábitat, investigaciones sobre el desarrollo humano. Bogotá: Panamericana Formas e impresos, (2004).

(2) Salas, J. (1998). Contra el hambre de vivienda, soluciones tecnológicas latinoamericanas. Bogotá: Escala, (1998).

(3) Vásquez Espí, M. (2001). Construcción e impacto sobre el ambiente: el caso de la tierra y otros materiales. Informes de la Construcción, 52(471): 29-43. https://doi.org/10.3989/ic.2001.v52.i471.681

(4) López, C., Ruiz, D., Jerez, S., Quiroga, P., Uribe, J. and Muñoz, E. (2007). Rehabilitación sísmica de muros de adobe de edificaciones monumentales mediante tensores de acero. Apuntes, 20(2): 304-317.

(5) INN (2013). Norma Chilena NCh3332. Estructuras. Intervención de construcciones patrimoniales de tierra cruda. Requisitos del proyecto estructural. Santiago, Chile: Instituto Nacional de Normalización.

(6) Ministerio de Vivienda Construcción y Saneamiento, República del Perú (2017). Norma Eo8o. Diseño y construcción con tierra reforzada. Lima: Ministerio de Vivienda, Construcción y Saneamiento.

(7) AIS (2004). Manual para la rehabilitación de viviendas construidas en adobe y tapia pisada. Bogotá: Asociación Colombiana de Ingeniería Sísmica.

(8) Papanikolau, A. and Taucer, F. (2004). Review of Non-Engineered Houses in Latin America with Reference to Building Practices and Self-Construction Projects, EUR 2119oEN. European Commission, Joint Research Centre. European Laboratory for Structural Assessment (ELSA). Retrieved from http://publications.jrc.ec.europa.eu/repository/bitstream/ JRC27945/EUR\%2021190\%20EN.pdf

(9) Yamin, L., Rodríguez, A., Fonseca, L., Reyes, J. and Phillips, C. (2003). Comportamiento sísmico y alternativas de rehabilitación de edificaciones en adobe y tapia pisada con base en modelos a escala reducida ensayados en mesa vibratoria. Revista de Ingeniería, 18: 175-190.

(10) Blondet, M., Vargas, J., Tarque, N. and Iwaki, C. (2011). Construcción sismorresistente en tierra: la gran experiencia contemporánea de la Pontificia Universidad Católica del Perú. Informes de la construcción, 63(523): 41-50. https://doi. org/10.3989/ic.10.017

(11) Yamín, L. E., Phillips, C., Reyes, J. C. and Ruíz, D. (2007). Estudios de vulnerabilidad sísmica, rehabilitación y refuerzo de casas en adobe y tapia pisada. Apuntes, 20(2): 286-303.

(12) Tolles, L. and Krawinkler, H. (1990). Seismic Studies on Small-Scale Models on Adobe Houses. John A. Blume Earthquake Engineering Center Technical Report Series No 91. Stanford University, (1990).

(13) Ruiz, D., López, C., Unigarro, S. and Domínguez, M. (2014). Seismic Rehabilitation of Sixteenth- and SeventeenthCentury Rammed Earth-Built Churches in the Andean Highlands: Field and Laboratory Study. Journal of Performance of Constructed Facilities, 29(6): 04014144. https://doi.org/10.1061/(ASCE)CF.1943-5509.0000605

(14) Ruiz, D., Silva, M., Cerón, L. and López, C. (2017). Seismic performance of rammed earth town halls reinforced with confinement wooden elements. Revista Ingeniería de Construcción, 32(2): 25-44. https://doi.org/10.4067/So71850732017000200003

(15) Silveira, D., Varum, H., Costa, A., Martins, T., Pereira, H. and Almeida, J. (2012). Mechanical properties of adobe bricks in ancient constructions. Construction and Building Materials, 28(1): 36-44. https://doi.org/10.1016/j.conbuildmat.2011.08.046

(16) Parisi, F., Asprone, D., Fenu, L. and Prota, A. (2015). Experimental characterization of Italian composite adobe bricks reinforced with straw fibers. Composite Structures, 122: 300-307. https://doi.org/10.1016/j.compstruct.2014.11.060

(17) Gómez, V, López, C., Ruiz, D. (2016). Rehabilitación sísmica de edificaciones históricas en tapia pisada: estudio de caso de capillas doctrineras reforzadas con malla de acero y madera de confinamiento. Informes de la Construcción, 68(541): e140. https://doi.org/10.3989/ic.15.017

(18) Varum, H., Figueiredo, A., Silveira, D., Martins, T., Costa, A. (2011). Investigaciones realizadas en la Universidad de Aveiro sobre caracterización mecánica de las construcciones existentes en adobe en Portugal y propuestas de rehabilitación y refuerzo. Resultados alcanzados. Informes de la Construcción, 63(523): 127-142. https://doi.org/10.3989/ ic.10.016

(19) Charleson, A. and Blondet, M. (2012). Seismic Reinforcement for Adobe Houses with Straps from Used Car Tires. Earthquake Spectra, 28(2): 511-530. https://doi.org/10.1193/1.4000014

(20) Illampas, R., Ioannou, I. and Charmpis, D. (2014). Adobe bricks under compression: Experimental investigation and derivation of stress-strain equation. Construction and Building Materials, 53: 83-90. https://doi.org/10.1016/j.conbuildmat.2013.11.103

(21) Harris, H. and Sabnis, G. (1999). Structural modeling and experimental techniques. Washington D.C.: CRC Press, (1999). 